\title{
Port-Hamiltonian Formulation of Systems With Memory
}

\author{
This paper investigates extending the properties of memory elements to model, \\ analyze, and simulate mechanical, electromechanical, hydromechanical, and \\ thermodynamic systems.
}

By Dimitri Jeltsema and Arnau Dòria-Cerezo

\begin{abstract}
In this paper, we consider memristors, meminductors, and memcapacitors and their properties as portHamiltonian systems. The port-Hamiltonian formalism naturally arises from network modeling of physical systems in a variety of domains. Exposing the relation between the energy storage, dissipation, and interconnection structure, this framework underscores the physics of the system. One of the strong aspects of the port-Hamiltonian formalism is that a power-preserving interconnection between port-Hamiltonian systems results in another port-Hamiltonian system with composite energy, dissipation, and interconnection structure. This feature can advantageously be used to model, analyze, and simulate networks consisting of complex interconnections of both conventional and memory circuit elements. Furthermore, the port-Hamiltonian formalism naturally extends the fundamental properties of the memory elements beyond the realm of electrical circuits.
\end{abstract}

KEYWORDS | Memory elements; memristive systems; memristor; port-based modeling; port-Hamiltonian systems

\section{INTRODUCTION AND MOTIVATION}

In the early 1970s, Chua [1] postulated the existence of a new basic electrical circuit element, called the memristor. The memristor, a contraction of memory and resis-

Manuscript received September 15, 2010; revised May 3, 2011; accepted July 25, 2011. Date of publication September 22, 2011; date of current version May 10, 2012. The work of A. Dòria-Cerezo was supported in part by the Spanish Government under Research Project DPI2010-15110.

D. Jeltsema is with the Delft Institute of Applied Mathematics, Delft University of Technology, 2628 CD Delft, The Netherlands (e-mail: d.jeltsema@tudelft.nl).

A. Dòria-Cerezo is with the Department of Electrical Engineering and the

Institute of Industrial and Control Engineering, Universitat Politècnica de Catalunya, 08800 Vilanova i la Geltrú, Spain (e-mail: arnau.doria@upc.edu).

Digital Object Identifier: 10.1109/JPROC.2011.2164169 tance that refers to a resistor with memory, completes the family of the well-known existing fundamental circuit elements: the resistor, the inductor, and the capacitor. Although a variety of physical devices, including thermistors, discharge tubes, Josephson junctions, and even ionic systems like the Hodgkin-Huxley model of a neuron, were shown to exhibit memristive effects [2], [3], a physical passive two-terminal memristive prototype could not be constructed until very recently scientists of HewlettPackard Laboratories announced its realization in [14]. Strukov et al. show that memristance naturally arises in nanoscale systems when electronic and atomic transports are coupled under an external bias voltage. In [6], the concept of memristance is extended to inductive and capacitive elements, called meminductors and memcapacitors.

One of the main reasons why the concept of memristance has not yet played a major role in modeling problems can most likely be explained from the fact that so far the majority of practical devices are reasonably well modeled by some (though often artificial) combination of standard modeling building blocks, like resistive, inductive, and capacitive elements, and their nonlinear and multiport versions. However, as nanoscale devices become more and more important and complex [2], it might be beneficial, and on the longer term even necessary, to enlarge our repertoire of modeling building blocks that establishes a closer connection between the mathematics and the observed physics.

In this contribution, we study memristive, meminductive, and memcapacitive behavior in the port-Hamiltonian modeling framework. Based on the mechanics formulation introduced by Sir W. R. Hamilton in the 19th century, the port-Hamiltonian formalism naturally arises from network modeling of physical systems in a variety of domains (e.g., electrical, mechanical, electromechanical, 
hydrodynamical, and thermodynamical). A strong aspect of the port-Hamiltonian formalism is that it underscores the physics of the systems by exposing the relation between the energy storage, dissipation, and interconnection structure. Furthermore, port-Hamiltonian systems are modular. This means that a power-preserving interconnection between port-Hamiltonian systems results in another portHamiltonian system with composite energy, dissipation, and interconnection structure. Based on this principle, complex, multidomain systems can be split into open subsystems. The subsystems are modeled individually and the resulting submodels can be stored in libraries. The modeling process is then performed in an iterative manner and the model of the overall system is constructed by interconnecting the submodels. This feature can advantageously be used to model, analyze, and simulate networks consisting of complex interconnections of both conventional and memory circuit elements.

The remainder of the paper is organized as follows. In Section II, we briefly characterize the port-Hamiltonian structure of an electrical circuit consisting of resistors, inductors, capacitors, and independent sources. Although the general structure of a port-Hamiltonian system possesses rich mathematical properties, we will only highlight those characteristics that are central in the expositions throughout the paper. The interested reader is referred to [17] for a basic introduction, and to [7] for a comprehensive summary of the developments of the port-Hamiltonian framework over the past two decades. Section III reviews the basic properties of the memristor, meminductor, and memcapacitor, followed by their characterization in the port-Hamiltonian framework in Section IV. In Section V, the fundamental properties of the memory elements are naturally extended beyond the realm of electrical circuits. Especially in the mechanical domain there exists a variety of simple devices that can serve as didactic examples to explain memristive, meminductive, and memcapacitive behavior.

\section{THE PORT-HAMILTONIAN FORMALISM}

The dynamical behavior of any electrical circuit is basically determined by three types of equations: those arising from Kirchhoff's voltage law, those arising from Kirchhoff's current law, and the constitutive relationships of the elements. In many cases, this leads to a large set of differential equations, often without any apparent structure, of the form $\dot{x}=f(x, u), y=g(x, u)$, where $u$ is a vector of inputs to the circuit, $x$ is a vector of state variables, and $y$ is a vector of designated outputs of interest. From a portHamiltonian perspective, an electrical circuit is considered as an interconnection of a port consisting of energy storing elements (inductors and capacitors), a port consisting of energy dissipating elements (resistors), and a port that represents the energy exchange with the environment (voltage and current sources). For a large class of circuits, this amounts to a system of differential equations of the form

$$
\begin{aligned}
& \dot{x}=[J(x)-D(x)] \frac{\partial H}{\partial x}(x)+B(x) u \\
& y=B^{T}(x) \frac{\partial H}{\partial x}(x)+M(x) u .
\end{aligned}
$$

As before, vectors $u, x$, and $y$ represent inputs, states, and outputs, respectively. However, the physical structure of the circuit is now reflected by the total energy $H(x)$ stored in the inductors and the capacitors, the symmetric resistive structure matrices $D(x)$ and $M(x)$ covering the energy dissipation in the resistors, and $B(x)$ describing the energy flows with the environment via the external ports. The power-preserving interconnections and elements such as transformers and gyrators are covered by the structure matrix $J(x)$, which is assumed to be skew-symmetric, that is, $J(x)=-J^{T}(x)$. System (1) is referred to as an inputstate-output port-Hamiltonian system with as Hamiltonian the total stored energy.

\section{A. Passivity}

A particularly appealing feature of a port-Hamiltonian system of the form (1) is that, because of skew-symmetry of $J(x)$, the energy flow of the circuit satisfies

$$
\dot{H}(x)=u^{T} y-\left[\frac{\partial H}{\partial x}(x)\right]^{T} D(x) \frac{\partial H}{\partial x}(x)-u^{T} M(x) u
$$

expressing that the power absorbed by the inductors and the capacitors equals the power supplied to the circuit via the external port minus the power dissipated by the resistors. Needless to say that (2) is just a restatement of Tellegen's theorem. Furthermore, if the resistive structure matrices $D(x) \succeq 0$ and $M(x) \succeq 0$ and $H(x)$ is bounded from below, the circuit satisfies the power-balance inequality $\dot{H}(x) \leq u^{T} y$, which states that the circuit cannot store more energy than it receives from the environment. In other words, the circuit is passive with respect to the supply rate $u^{T} y$ and storage function $H(x)$. In the special case that $D(x)=0$ and $M(x)=0$, we have $\dot{H}(x)=u^{T} y$, implying that the circuit is lossless.

\section{B. Resistors, Inductors, and Capacitors as Port-Hamiltonian Input-Output Systems}

Since electronics was developed, engineers designed circuits using combinations of three basic elements: resistors, inductors, and capacitors. From a mathematical perspective, the behavior of each of these elements, whether linear or nonlinear, is described by relationships between two of the four fundamental electrical variables: 


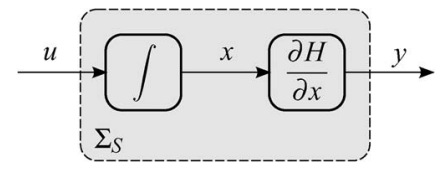

Fig. 1. General structure of an energy storage element.

voltage, current, charge, and flux-linkage (hereafter simply referred to as flux). A resistor is described by the relationship of current and voltage; an inductor by that of current and flux, and a capacitor by that of voltage and charge. Hence, from a port-Hamiltonian perspective, a resistor can then be considered as a static input-output system of the form

$$
\Sigma_{R}: y=\hat{y}(u)
$$

which is just a generalized version of Ohm's law in which $u$ and $y$ represent either voltage and current (impedance form) or current and voltage (admittance form), respectively. In the linear case, the resistive port (3) reduces to either $y=R u$ or $y=G u$, with $G=R^{-1}$ the inverse resistance or conductance. Note that for a passive resistor the dissipated power $u y \geq 0$.

On the other hand, from a port-Hamiltonian perspective, the structure of all energy storage elements is identical. Assuming that the constitutive relationships can be written in the form $y=\hat{y}(x)$, a storage element can be characterized by an input $u$, an output $y$, a physical state $x$, and a energy function

$$
H(x)=\int_{x_{0}}^{x} \hat{y}(s) d s
$$

with $x_{0}$ some initial state, as depicted in Fig. 1. The associated state equations of the element define the portHamiltonian system

$$
\Sigma_{S}:\left\{\begin{array}{l}
\dot{x}=u \\
y=\frac{\partial H}{\partial x}(x)
\end{array}\right.
$$

which, depending on the nature of the input, state, and output, represents either an inductor or a capacitor. Indeed, if $u$ is a voltage, then, by Faraday's law, $x$ represents a flux, and, as a consequence, $y$ represents the current through an inductor. The associated magnetic energy is defined by the integral of current with respect to the flux. Dually, the state equations of a capacitor are obtained by selecting as input a current, resulting in a charge as state variable, and consequently a voltage as output. The associated electric energy is defined by the integral of voltage with respect to charge. Note that in both cases the absorbed power is characterized by the rate of change of energy $\dot{H}(x)=u y$ (= voltage $\times$ current, or vice versa). These properties are exemplified in more detail in Section II-D.

\section{Modularity}

It is argued before that from a port-Hamiltonian perspective an electrical circuit can be considered as a powerpreserving interconnection of an energy storage port, a dissipation port, and an external interaction port. In a similar fashion, we can also interconnect several portHamiltonian systems. For example, the interconnection of the two port-Hamiltonian systems

$$
\begin{aligned}
& \dot{x}_{i}=\left(J_{i}-D_{i}\right) \frac{\partial H_{i}}{\partial x_{i}}\left(x_{i}\right)+B_{i} u_{i} \\
& y_{i}=B_{i}^{T} \frac{\partial H_{i}}{\partial x_{i}}\left(x_{i}\right), \quad i=1,2
\end{aligned}
$$

via the feedback interconnection $u_{1}=-y_{2}, u_{2}=y_{1}$ (which is power-preserving since $u_{1} y_{1}+u_{2} y_{2}=0$ ), yields another port-Hamiltonian system

$$
\left(\begin{array}{c}
\dot{x}_{1} \\
\dot{x}_{2}
\end{array}\right)=\left(\begin{array}{cc}
J_{1}-D_{1} & -B_{1} B_{2}^{T} \\
B_{2} B_{1}^{T} & J_{2}-D_{2}
\end{array}\right)\left(\begin{array}{l}
\frac{\partial H_{1}}{\partial x_{1}}\left(x_{1}\right) \\
\frac{\partial H_{2}}{\partial x_{2}}\left(x_{2}\right)
\end{array}\right)
$$

with total Hamiltonian $H_{1}\left(x_{1}\right)+H_{2}\left(x_{2}\right)$.

\section{Example: Classical Josephson Junction Circuit Model}

Consider the classical circuit model for a Josephson junction shown in Fig. 2. The circuit consists of a parallel connection of a linear resistor $R$, a linear capacitor $C$, and a flux-controlled nonlinear inductor described by the constitutive relationship $I_{L}=I_{o} \sin \left(k \phi_{L}\right)$, where $I_{o}$ is a device parameter and $k=4 \pi \epsilon / \bar{h}$, with $\epsilon$ and $\bar{h}$ denoting the electron charge and Planck's constant, respectively.

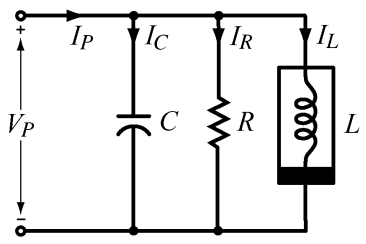

Fig. 2. Classical Josephson junction circuit model. 
From a port-Hamiltonian perspective, the circuit is considered as a power-preserving interconnection of energy storing elements, energy dissipating elements, and energy supplying elements.

The inductor stores magnetic energy due to accumulation of magnetic flux, that is

$$
H_{L}\left(\phi_{L}\right)=\int_{0}^{\phi_{L}} I_{o} \sin (k \phi) d \phi=-\frac{I_{o}}{k} \cos \left(k \phi_{L}\right)+c .
$$

According to (4), the inductor is then completely described by the port-Hamiltonian subsystem

$$
\Sigma_{L}:\left\{\begin{array}{l}
\dot{\phi}_{L}=V_{L} \\
I_{L}=\frac{\partial H_{L}}{\partial \phi_{L}}\left(\phi_{L}\right)
\end{array}\right.
$$

In the light of Fig. $1, V_{L}$ acts as the input, $\phi_{L}$ as the state, and $I_{L}$ as the output. Note that the power absorbed by the inductor is characterized by the rate of change in the stored magnetic energy $\dot{H}_{L}\left(\phi_{L}\right)=V_{L} I_{L}$, where $\left(V_{L}, I_{L}\right)$ constitutes a power conjugate input-output pair.

In a similar fashion, the capacitor stores electric energy due to accumulation of electric charge. Hence, integrating the voltage across the capacitor with respect to the charge, with $V_{C}=(1 / C) q_{C}$, yields the quantity $H_{C}\left(q_{C}\right)=(1 / 2 C) q_{C}^{2}$, which, together with the property that current equals the rate of change of charge, defines the port-Hamiltonian subsystem

$$
\Sigma_{C}:\left\{\begin{array}{l}
\dot{q}_{C}=I_{C} \\
V_{C}=\frac{\partial H_{C}}{\partial q_{C}}\left(q_{C}\right)
\end{array}\right.
$$

and $\dot{H}_{C}\left(q_{C}\right)=I_{C} V_{C}$.

Energy dissipation is due to the resistor in the circuit and is characterized by a static relation between the associated voltage and current. Since the resistor is linear, two representations exist: $V_{R}=R I_{R}$ or $I_{R}=G V_{R}$, with $G=R^{-1}$. Note that with $R \geq 0$, the power dissipated by the resistive subsystem $\Sigma_{R}$ satisfies $I_{R} V_{R} \geq 0$.

The remaining port, with port variables $\left(I_{P}, V_{P}\right)$, denotes the interaction port of the system, modeling its interaction with the environment. The power supplied to the interaction port equals $V_{P} I_{P}$, which in the following is referred to as the supply rate.

Finally, the part of the circuit which neither involves energy storage nor energy dissipation or energy supply, is defined by linear relations between the voltages across the ports and the currents into the ports that satisfy Kirchhoff's current and voltage laws. With the sign conventions as in

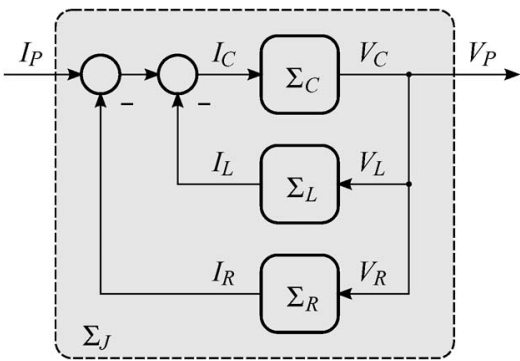

Fig. 3. Classical Josephson junction circuit model as a feedback interconnection of port-Hamiltonian subsystems.

Fig. 2, these relations are given by $I_{P}-I_{R}-I_{L}-I_{C}=0$ and $V_{R}=V_{L}=V_{C}=V_{P}$, respectively. Combining the latter with the port characterizations given above, yields [compare with (1)]

$$
\begin{aligned}
\left(\begin{array}{c}
\dot{\phi}_{L} \\
\dot{q}_{C}
\end{array}\right) & =\left[\left(\begin{array}{cc}
0 & 1 \\
-1 & 0
\end{array}\right)-\left(\begin{array}{ll}
0 & 0 \\
0 & G
\end{array}\right)\right]\left(\begin{array}{l}
\frac{\partial H_{S}}{\partial \phi_{L}} \\
\frac{\partial H_{S}}{\partial q_{C}}
\end{array}\right)+\left(\begin{array}{l}
0 \\
1
\end{array}\right) I_{P} \\
V_{P} & =\left(\begin{array}{ll}
0 & 1
\end{array}\right)\left(\begin{array}{l}
\frac{\partial H_{S}}{\partial \phi_{L}} \\
\frac{\partial H_{S}}{\partial q_{C}}
\end{array}\right)
\end{aligned}
$$

where $H_{S}\left(\phi_{L}, q_{C}\right)=H_{L}\left(\phi_{L}\right)+H_{C}\left(q_{C}\right)$ is the total stored energy. The interconnected circuit may thus be considered as a port-Hamiltonian system with input $I_{P}$, states $\left(\phi_{L}, q_{C}\right)$, output $V_{P}$, and Hamiltonian $H_{S}\left(\phi_{L}, q_{C}\right)$; see Fig. 3.

Differentiating the total stored energy with respect to time, it is readily observed that

$$
\dot{H}_{S}\left(\phi_{L}, q_{C}\right)=I_{P} V_{P}-G\left[\frac{\partial H_{S}}{\partial q_{C}}\left(\phi_{L}, q_{C}\right)\right]^{2}
$$

which is just Tellegen's theorem restating the powerpreserving property that $I_{L} V_{L}+I_{C} V_{C}+I_{R} V_{R}=I_{P} V_{P}$. Furthermore, since $G \geq 0$ and $H_{S}\left(\phi_{L}, q_{C}\right)$ is bounded from below, $\dot{H}_{S}\left(\phi_{\mathrm{L}}, q_{C}\right) \leq I_{P} V_{P}$, yielding that the Josephson junction circuit model is passive with respect to the externally suppled power and the total stored energy.

\section{MEMORY ELEMENTS}

\section{A. The Memristor}

In Section II-B, we have seen that the three basic circuit elements are described by a combination of two out 
of the four fundamental variables. This leaves one relationship undefined, namely the one between charge and flux. As argued by Chua in the early 1970s [1], a fourth element should be added to complete the symmetry. He coined this "missing element" the memristor, referring to a resistor with memory. The memory aspect stems from the fact that a memristor "remembers" the amount of current that has passed through it together with the total applied voltage. More specifically, if $q$ denotes the charge and $\phi$ denotes the flux, then a charge-modulated memristor is defined by the constitutive relationship $\phi=\hat{\phi}(q)$. Since flux is defined by the time integral of voltage $V$ (like in Faraday's law), and charge is the time integral of current $I$, or equivalently, $V=\dot{\phi}$ and $I=\dot{q}$, we obtain

$$
V=R_{M}(q) I
$$

where $R_{M}(q):=d \phi / d q$ is the incremental memristance.

Observe that (6) is just a charge-modulated version of Ohm's law. It is important to realize that for the very special case that the constitutive relation is linear, that is, when the incremental memristance $R_{M}(q)$ is constant, a memristor becomes an ordinary resistor. Hence, memristors are only relevant in nonlinear circuits, which may account in part for its neglect in linear network and systems theory. Furthermore, it is directly noticed from (6) that $V \equiv 0$ whenever $I \equiv 0$, regardless of $q$, which incorporates the memory effect. This characteristic feature is the so-called no energy discharge property [2], [6], which is related to the fact that, unlike an inductor or a capacitor, a memristor does not store energy.

Remark 1: Note that (6) is the definition of a memristor in impedance form. The admittance form $I=G_{M}(\phi) V$, with incremental memductance $G_{M}(\phi):=d q / d \phi$, is obtained by starting from a constitutive relationship $q=\hat{q}(\phi)$.

\section{B. Meminductors and Memcapacitors}

In addition to memristors, two new types of memory elements, called meminductors and memcapacitors, are introduced in [6]. Let $\sigma$ and $\rho$ denote the time integrals of charge and flux, respectively, or equivalently, $\dot{\sigma}=q$ and $\dot{\rho}=\phi$. Then, in a similar fashion as the memristor, relationships can be defined between charge, flux, integrated charge, and integrated flux. Indeed, consider the relationship $\rho=\hat{\rho}(q)$. Differentiation of the latter with respect to time yields

$$
\phi=L_{M}(q) I
$$

Since $L_{M}(q):=d \rho / d q$ relates flux with current, its values clearly correspond to the units of inductance. Hence, the

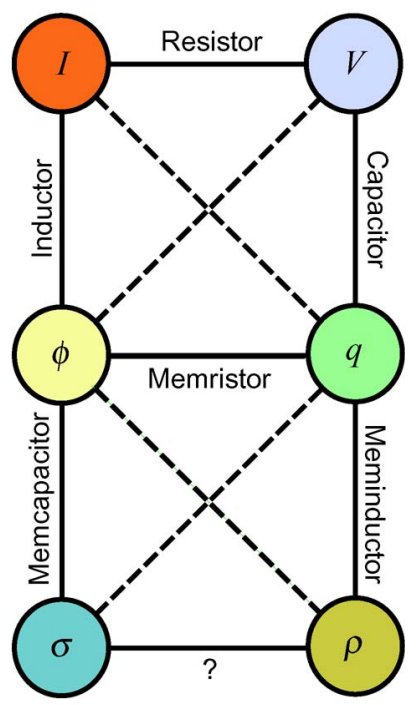

Fig. 4. The three basic electrical elements and their memory counterparts: memristor, meminductor, and memcapacitor. The dynamical relationships $I=\dot{q}$ and $V=\dot{\phi}$ together with the newly introduced relations $\dot{\sigma}=q$ and $\dot{\rho}=\phi$ are represented by the dashed diagonal lines.

latter defines the incremental inductance of a chargemodulated meminductor. Bearing in mind that charge is the time integral of current, the memory aspect of a charge-modulated meminductor stems from the fact that it "remembers" the amount of current that has passed through it. The energy storage capabilities are discussed in Section IV-D.

Dually, a flux-modulated memcapacitor is defined by $\sigma=\hat{\sigma}(\phi)$, which, after differentiation with respect to time, yields

$$
q=C_{M}(\phi) V
$$

where $C_{M}(\phi):=d \sigma / d \phi$ denotes the incremental capacitance. The memory aspect of a flux-modulated memcapacitor stems from the fact that it "remembers" the amount of voltage that has been applied to it.

A meminductor (resp. memcapacitor) that depends on the history of its flux (resp. charge) can be formulated by starting from a constitutive relationship of the form $q=\hat{q}(\rho)$ (resp. $\phi=\hat{\phi}(\sigma))$. The results are summarized in Fig. 4.

\section{PORT-HAMILTONIAN MEMORY SYSTEMS}

\section{A. Memristive Ports and the Null-Hamiltonian}

In the port-Hamiltonian framework, the fundamental property that a memristor cannot store any energy 
(recall the "no energy discharge property" discussed in Section III-A) is emphasized by the fact that we can associate with (6) a so-called null-Hamiltonian. Indeed, comparison of (6), together with $\dot{q}=I$, with the port-Hamiltonian system (1) reveals that by letting $u=I, x=q, y=V$, $J(x)=0, \quad D(x)=0, \quad B(x)=1$, and $M(x)=R_{M}(q)$, we may characterize a charge-modulated memristor as

$$
\dot{x}=u \quad y=\frac{\partial H}{\partial x}(x)+M(x) u
$$

where $H(x) \equiv 0$ represents the null-Hamiltonian. Furthermore, since $\dot{H}(x)=(\partial H / \partial x)(x) \dot{x} \equiv 0$, we have $u(y-M(x) u) \equiv 0$, which in turn implies that the power occurring at the memristive port is given by $u y=M(x) u^{2}$. Hence, a memristive port is passive if and only if its memristance is nonnegative.

Remark 2: Note that the port-Hamiltonian structure of a flux-modulated memristor (see Remark 1) is obtained by letting $u=V, x=\phi, y=I$, and $M(x)=G_{M}(\phi)$.

\section{B. Example: The Josephson Junction Revisited}

As discussed in [2], a more rigorous quantum mechanical analysis of the junction dynamics reveals the presence of an additional small current component that can be approximated by $I_{M}=\gamma \cos \left(k_{0} \phi_{M}\right) V_{M}$, for some constants $\gamma, k_{0}$. Obviously, the latter can be associated with a fluxmodulated memristor having a constitutive relationship $q_{M}=\left(\gamma / k_{0}\right) \sin \left(k_{0} \phi_{M}\right)$. Hence, a more realistic circuit model for a Josephson junction consists of a parallel connection of a resistor, inductor, capacitor, and a memristor; see Fig. 5(a). From a port-Hamiltonian perspective, the



(a)

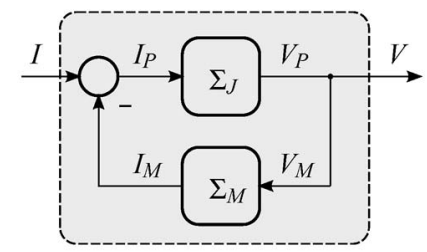

(b)

Fig. 5. Modified Josephson junction: (a) circuit model; (b) interpretation as a feedback interconnection of two port-Hamiltonian systems. extension of the classical circuit model discussed in Section II-D with a memristor can be accomplished by following the ideas outlined in Section II-C and considering a negative feedback interconnection of the two portHamiltonian systems (5) and

$$
\Sigma_{M}:\left\{\begin{array}{l}
\dot{\phi}_{M}=V_{M} \\
I_{M}=\frac{\partial H_{M}}{\partial \phi_{M}}\left(\phi_{M}\right)+G_{M}\left(\phi_{M}\right) V_{M}
\end{array}\right.
$$

with $G_{M}\left(\phi_{M}\right):=\gamma \cos \left(k_{o} \phi_{M}\right)$ and $H_{M}\left(\phi_{M}\right) \equiv 0$, via $I_{P}=-I_{M}+I$ and $V_{P}=V_{M}=V$, where $I$ and $V$ denote a new external input-ouput pair; see Fig. 5(b). The resulting port-Hamiltonian system becomes

$$
\begin{aligned}
\left(\begin{array}{c}
\dot{\phi}_{L} \\
\dot{q}_{C} \\
\dot{\phi}_{M}
\end{array}\right) & =\left(\begin{array}{ccc}
0 & 1 & 0 \\
-1 & -G-G_{M}\left(\phi_{M}\right) & -1 \\
0 & 1 & 0
\end{array}\right)\left(\begin{array}{c}
\frac{\partial H}{\partial \phi_{L}} \\
\frac{\partial H}{\partial q_{C}} \\
\frac{\partial H}{\partial \phi_{M}}
\end{array}\right)+\left(\begin{array}{l}
0 \\
1 \\
0
\end{array}\right) I \\
V & =\left(\begin{array}{lll}
0 & 1 & 0
\end{array}\right)\left(\begin{array}{c}
\frac{\partial H}{\partial \phi_{L}} \\
\frac{\partial H}{\partial q_{C}} \\
\frac{\partial H}{\partial \phi_{M}}
\end{array}\right)
\end{aligned}
$$

with total stored energy $H\left(\phi_{L}, q_{C}, \phi_{M}\right)=H_{S}\left(\phi_{L}, q_{C}\right)+$ $H_{M}\left(\phi_{M}\right)$

\section{Order of Complexity}

The addition of the memristive port yields that the dimension of the total state space is in general increased. Consequently, in addition to the initial values of the state variables associated to the storage elements, also the initial values of the memristors should be specified in order to find a complete solution of the port-Hamiltonian systems presented above. For example, the order of complexity of the improved Josephson junction circuit model is three since three initial conditions are needed to solve the system. In general, this means that the order of complexity of a port-Hamiltonian system with memristive dissipation is determined by $n=n_{S}+n_{M}$, where $n_{S}$ denotes the number of independent energy storage elements and $n_{M}$ the number of independent memristive elements.

\section{Memstorage Ports}

Let us now consider the meminductor and the memcapacitor. In Section III, we have seen that these memory 
elements share many of the characteristics of a memristor, but with a fundamental difference: they do store energy. Indeed, the energy storage capability of a charge-modulated meminductor (7) becomes apparent by writing its dynamics in the form

$$
\begin{aligned}
& \dot{\phi}=V \\
& \dot{q}=L_{M}^{-1}(q) \phi
\end{aligned}
$$

where it is readily observed that $V \equiv 0$ does not imply $\phi \equiv 0$, and thus, in general, $\dot{q}=I \neq 0$ whenever $V=0$. Since a meminductor behaves as a linear inductor that is modulated by its charge, the stored energy is a quadratic function of the form $H(\phi, q)=(1 / 2) L_{M}^{-1}(q) \phi^{2}$. Clearly, the flux is related with energy storage, whereas the charge incorporates the memory effect. Furthermore, the rate of change in energy along the solutions of (10) yields the power balance

$$
\begin{aligned}
\dot{H}(\phi, q) & =\frac{\partial H}{\partial \phi}(\phi, q) \dot{\phi}+\frac{\partial H}{\partial q}(\phi, q) \dot{q} \\
& =L_{M}^{-1}(q) \phi \dot{\phi}-\frac{1}{2} \frac{d L_{M}}{d q}(q)\left[L_{M}^{-1}(q) \phi\right]^{2} \dot{q} \\
& =I V-\frac{1}{2} \dot{L}_{M}(q)\left[\frac{\partial H}{\partial \phi}(\phi, q)\right]^{2}
\end{aligned}
$$

expressing that the power absorbed by the meminductor equals the power supplied to it minus the power gained or lost due the change in the inductance.

Comparison of (11) to the power balance (2) suggests that the underlying port-Hamiltonian structure of the meminductor (10) is simply given by

$$
\begin{aligned}
& \dot{x}=[J-D(\cdot)] \frac{\partial H}{\partial x}(x)+B u \\
& y=B^{T} \frac{\partial H}{\partial x}(x)
\end{aligned}
$$

where $u=V$ denotes the input, $x=\operatorname{col}(\phi, q)$ denotes the state, and $y=I$ denotes the associated output. The remaining physical properties are explicitly reflected by the structure matrices

$$
J=\left(\begin{array}{cc}
0 & -1 \\
1 & 0
\end{array}\right) \quad D(\cdot)=\frac{1}{2}\left(\begin{array}{cc}
\dot{L}_{M}(q) & 0 \\
0 & 0
\end{array}\right) \quad B=\left(\begin{array}{l}
1 \\
0
\end{array}\right)
$$

and the Hamiltonian $H(x)=H(\phi, q)$ as given above.

The port-Hamiltonian structure of the memcapacitor (8) is obtained from (12) by reversing the input, the output, and the sequence of the state variables, letting $H(q, \phi)=(1 / 2) C_{M}^{-1}(\phi) q^{2}$, and changing the dissipative structure matrix to

$$
D(\cdot)=\frac{1}{2}\left(\begin{array}{cc}
\dot{C}_{M}(\phi) & 0 \\
0 & 0
\end{array}\right)
$$

\section{E. Passivity and Losslessness}

Although the "dissipation" term $(1 / 2) \dot{L}_{M}(q)$ in (11) suggests that a meminductor is lossy (resp. active) when inductance is lost (resp. gained), that is, when $\dot{L}_{M}(q)<0$ (resp. $\dot{L}_{M}(q)>0$ ), the element is in fact passive and lossless under the condition that its meminductance is nonnegative. Indeed, reconsidering (11), we observe that the power balance can be decomposed into two parts: a net energy exchange rate and a net energy storage rate $I V=(\partial H / \partial \phi)(\phi, q) \dot{\phi}$, which in turn implies that

$$
\int_{0}^{T} I(t) V(t) d t=\int_{0}^{T} L_{M}^{-1}(q(t)) \phi(t) d \phi(t) \geq 0
$$

if, and only if, $L_{M}(q) \geq 0$, for all $q$; see also [4]

Likewise, a memcapacitor is passive and lossless if, and only if, its memcapacitance is nonnegative, that is, $C_{M}(\phi) \geq 0$, for all $\phi$

\section{MECHANICAL SYSTEMS AND BEYOND}

In science and engineering, the ideas and concepts developed in one branch of science and engineering are often transferred to other branches. One approach to transferring ideas and concepts is by the use of analogies. In the previous developments, we have seen that an electrical circuit can be characterized by interconnections between energy storage elements, energy dissipating elements, and the environment. This basic idea holds in any physical domain. Next to the electrical domain, the most important physical domains are the mechanical, hydraulic, and thermic domains. One remarkable feature of the storage elements in each domain is that their structure is identical. In Section II-B, we have already seen that, although being dual elements, an inductor and a capacitor can both be characterized by the port-Hamiltonian representation (4) and structure shown in Fig. 1. From this perspective, it is natural to discriminate among domains depending on the kind of energy that a certain part of the system can store and split the usual physical domains into two subdomains. Each subdomain is then associated to only one type of storage. Consequently, we do not speak of electrical, mechanical, or hydraulic domains, but of electric and magnetic, mechanical kinetic and mechanical potential, or 
Table 1 Domain Classification in Port-Hamiltonian Framework: (a) Energy Storage. In Contrast to Common Port-Based Modeling Approaches, Such as the Standard Bondgraph Formalism [7] or Classical Energy- and Power-Based Approaches [9], the Port-Hamiltonian Framework Uses Only One Type of Storage. The Subdomain Classification Is Based on the Generalized Bond Graph (GBG) Framework Introduced in [7], Where $u$ Is Referred to as the Flow (Rate of Change of State) and $y$ as the Effort (Equilibrium-Determining Variable). Note That in Each Domain the Product uy Equals Power. (b) Generalization of the Memory State Variables

\begin{tabular}{|c|c|c|c|c|}
\hline physical subdomain & input $u$ & output $y$ & storage state $x_{S}$ & memory state $x_{M}$ \\
\hline electric & current & voltage & charge & flux linkage \\
\hline magnetic & voltage & current & flux linkage & charge \\
\hline potential translation & velocity & force & displacement & momentum \\
\hline kinetic translation & force & velocity & momentum & displacement \\
\hline potential rotation & angular velocity & torque & angular displacement & angular momentum \\
\hline kinetic rotation & torque & angular velocity & angular momentum & angular displacement \\
\hline potential hydraulic & volume flow & pressure & volume & flow tube momentum \\
\hline kinetic hydraulic & pressure & volume flow & flow tube momentum & volume \\
\hline thermal & entropy flow & temperature & entropy & integrated temperature \\
\hline \multicolumn{4}{|c|}{ (a) } & (b) \\
\hline
\end{tabular}

hydraulic kinetic and hydraulic potential subdomains. The thermic domain is the only one that has no dual subdomains, which is related to the irreversible transformation of energy in thermodynamics [7]. Table 1(a) shows a complete overview. Note that the resistive elements in each subdomain are simply represented by relationships between $u$ and $y$ of the form (3).

By considering only one type of storage, the state variables associated to each subdomain are all treated at the same footing. Consequently, the memory elements and their port-Hamiltonian representations can easily be generalized by considering the duals of the storage variables of Table 1(a). These dual variables constitute the memory states and are simply the time integrals of the output variables; see Table 1(b). Hence, the generic multidomain forms of the memristor and the memstorage elements are given by (9) and (12), respectively, whereas the associated input, state, and output variables of the storage elements can be readily obtained from the table.

Remark 3: Historically, the first attempt to relate mechanical and electrical systems was due to James Clerk Maxwell and Lord Kelvin in the 19th century by using the similarity between force and voltage, as is also apparent from the early use of the term electromotive force (emf). This force-voltage analogy implies that a mechanical mass is analogous to an electrical inductor. Once the forcevoltage analogy had been established, some scientists started to address some of its limitations. These limitations led to the alternative force-current analogy, which implies that a mechanical mass is analogous to an electrical capacitor. An advantage of considering only one type of storage is that the concept of mechanical force has no unique meaning as it may play the role of an "input" (rate of change of state or flow) in the kinetic domain or an "output" (equilibrium-determining variable or effort) in the potential domain. Hence, from a port-Hamiltonian perspective, the discussion which analogy-when it exists-is superior is left a nonissue.

\section{A. Mechanical Memristance}

Traditionally, the three basic building blocks to model a mechanical system are mass (or, alternatively, the twoterminal inerter [13]), spring, and damper. Mass has a central role in determining the dynamical behavior of bodies, a spring is used to represent the stiffness of a body, and a damper or dashpot is defined as a mechanical device which resists motion via friction. In contrast to a mass or a spring, where the net energy is not lost but rather stored as kinetic or potential energy, respectively, a damper generates heat that is dissipated into the environment. From a mathematical perspective, the behavior of each of these building blocks is described by a relationship between two of the four basic mechanical variables of Table 1(a): force, velocity, momentum, and displacement. A damper is described by a relationship between force and velocity; a mass by that of velocity and momentum; and a spring by that of force and position. According to Table 1(a), a mechanical memristor is then formally defined by a relationship between momentum and displacement. The simplest physical example of a mechanical memristor is a tapered dashpot shown in Fig. 6. This type of dashpot is a mechanical resistor whose resistance (that is, its friction coefficient) depends on the relative displacement of its terminals. Although a physical electrical passive twoterminal memristive device was constructed only recently [14], a tapered dashpot was already brought forward in the early 1970s in [11]. An example of a mechanical system

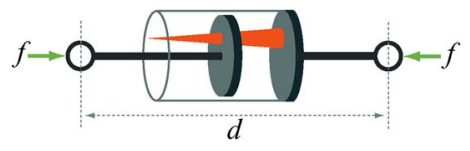

Fig. 6. Example of a tapered dashpot. The friction coefficient depends on the displacement $d$. In practice, the taper is achieved by a conical pin passing through an orifice in the piston. The shape of the pin can be machined to produce any desired memristive relation between displacement and momentum. 


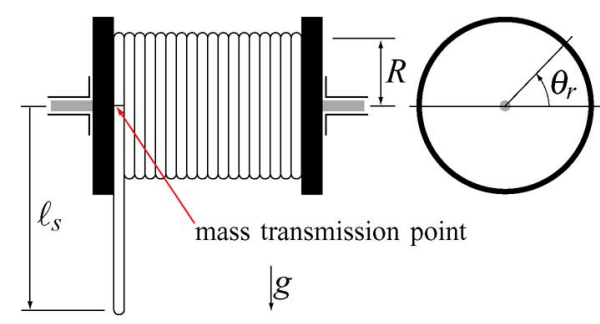

Fig. 7. A cable-reel system.

with a tapered dashpot in port-Hamiltonian form is presented in [10].

\section{B. Mechanical Memstorage}

Consider the elementary problem of a heavy cable that is deployed from a reel as illustrated in Fig. 7. Physically, the subsystem consisting of the reel and the wounded part of the cable can be separated at the indicated mass transmission port and can be considered as a positiondependent moment of inertia [8] of the form $I\left(\theta_{r}\right)=I_{0}+$ $\mu R^{3} \theta_{r}$, where $\theta_{r} \geq 0$ denotes the angular displacement, $R$ and $I_{o}$ denote the radius and the moment of inertia around the axis of rotation of the empty reel, respectively, and $\mu$ denotes the mass per unit length. Since a moment of inertia is defined by a relationship between angular momentum $p_{r}$ and angular velocity $\omega_{r}=\dot{\theta}_{r}$, we have $p_{r}=$ $I\left(\theta_{r}\right) \omega_{r}$. To obtain some insights in the behavior, let us actuate the system with a sinusoidal angular velocity $\omega_{r}=$ $A \sin \left(2 \pi f_{T} t\right)$ and compare the position-dependent moment of inertia for different values of $\mu$. The results are shown in Fig. 8. Note that only for $\mu=0$ the relationship between the angular momentum and the angular velocity is linear,

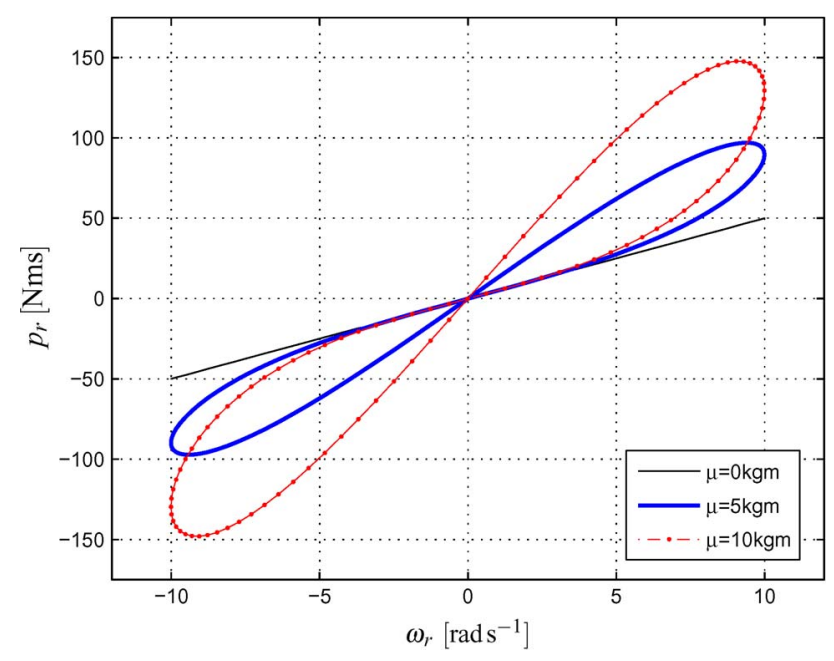

Fig. 8. Simulation of the angular momentum versus angular velocity curve of a position-dependent moment of inertia. The system parameters used are $I_{O}=5 \mathrm{~kg} \mathrm{~m}^{2}, R=1 \mathrm{~m}, A=10 \mathrm{rad} \mathrm{s}^{-1}$, and $f_{T}=2 \mathrm{~Hz}$. while for $\mu>0$ a pinched hysteresis loop appears. Such pinched hysteresis loops have been identified in the electrical domain as the fingerprints for the circuit elements with memory discussed in Section III. Indeed, suppose that we consider, instead of a relationship between angular momentum and angular velocity, a constitutive relationship between integrated angular momentum and angular displacement $\rho_{r}=I_{o} \theta_{r}+(1 / 2) \mu R^{3} \theta_{r}^{2}=\hat{\rho}_{r}\left(\theta_{r}\right)$, with $\dot{\rho}_{r}=$ $p_{r}$. Now, differentiating the latter with respect to time yields $p_{r}=\left(d \hat{\rho}_{r} / d \theta_{r}\right)\left(\theta_{r}\right) \omega_{r}$, which, by defining $I\left(\theta_{r}\right):=$ $d \hat{\rho}_{r}\left(\theta_{r}\right) / d \theta_{r}$, precisely coincides with our earlier definition. The main difference, however, is that $p_{r}=I\left(\theta_{r}\right) \omega_{r}$ is rather a consequence of the single-valued relationship $\rho=\hat{\rho}_{r}\left(\theta_{r}\right)$, and not vice versa. For these reasons, it is natural to consider the cable-reel subsystem as a memory-mass element belonging to the kinetic rotation domain. According to Table 1, the state consists of the angular momentum associated to the storage of energy and the angular displacement incorporating the memory effect. See [8] for more details and a port-Hamiltonian description of the cablereel system.

\section{CONCLUDING REMARKS}

We have extended the port-Hamiltonian formalism with the inclusion of memristive, meminductive, and memcapacitive elements and briefly discussed their multidomain generalizations. Although we have only highlighted the most basic characteristics and properties, it is our belief that the port-Hamiltonian formalism provides a solid and natural framework for modeling and analysis. Among the many questions and problems that remain open, we remark the following.

- The extension of the port-Hamiltonian framework to include memristive systems, which generalizes the basic memristor concept to a much broader class of dynamical systems [3], is discussed in [10]. The next step is to study under what conditions the meminductive and memcapacitive systems proposed in [6] can be captured in the portHamiltonian formalism.

- The memristor, meminductor, and memcapacitor are actually special cases of a complete family of socalled higher order elements [2], [4]. Hamiltonian descriptions of higher order elements are reported in [5] and [16]. However, these works are mainly concerned with linear higher order elements and lack the motivation of the theory with clear and motivating practical examples. Furthermore, the Hamiltonian descriptions developed in [5] and [16] are only valid for a very restricted class of nonlinear higher order elements, excluding the memristor, meminductor, and memcapacitor. Current research is devoted to the classification and identification of the higher elements from both electrical and mechanical perspectives. 
- We have discussed two simple examples of mechanical memory elements: a tapered dashpot (position-dependent damper) and the cable-reel (position-dependent mass). Physical examples of memory springs are most likely to be found in elastic materials. The fingerprint for such a device is a pinched hysteresis loop in the force-displacement plane. Some preliminary literature research reveals piano hammers as possible candidate memory spring devices [15]. Examples of memory analogs in the hydraulic and thermic domains are to be identified.
- Apart from enlarging our repertoire of modeling building blocks, the inclusion of memory elements in the existing port-Hamiltonian formalism possibly opens up new ideas for controller synthesis and design. Moreover, some preliminary research shows that memristors are capable of learning [12]. These properties seem to be very interesting from an adaptive, predictive, or gain scheduling control point of view. Similar phenomena are expected from meminductors and memcapacitors.

\section{REFERENCES}

[1] L. O. Chua, "Memristor, the missing circuit element," IEEE Trans. Circuit Theory, vol. 18, no. 5, pp. 507-519, Sep. 1971.

[2] L. O. Chua, "Nonlinear circuit foundations for nanodevices - Part I: The four-element torus," Proc. IEEE, vol. 91, no. 11, pp. 1830-1859, Nov. 2003

[3] L. O. Chua and S. M. Kang, "Memristive devices and systems," Proc. IEEE, vol. 64, no. 2, pp. 209-223, Feb. 1976.

[4] L. O. Chua and E. W. Szeto, "High-order nonlinear circuit elements: Circuit-theoretic properties," Int. J. Circuit Theory Appl., vol. 11, no. 2, pp. 187-206, Apr. 1983.

[5] C. Civelek, "Mathematical modelling of rotational mechanical elements of higher order and their characteristics," Math. Comput. Model., vol. 43, no. 7-8, pp. 957-964, Apr. 2006.

[6] M. Di Ventra, Y. V. Pershin, and L. O. Chua, "Circuit elements with memory: Memristors, memcapacitors, and meminductors," Proc. IEEE, vol. 97, no. 10, pp. 1717-1724, Oct. 2009.
[7] V. Duindam, A. Macchelli, S. Stramigioli, and H. Bruyninckx, Eds., Modeling and Control of Complex Physical Systems: The Port-Hamiltonian Approach. New York: Springer-Verlag, 2009.

[8] D. Jeltsema and A. Dòria-Cerezo, "Mechanical memory elements: Modeling of systems with position-dependent mass revisited," in Proc. IEEE Conf. Decision Control, Dec. 2010, pp. 3511-3516.

[9] D. Jeltsema and J. M. A. Scherpen, "Multi-domain modeling of nonlinear networks and systems: Energy- and power-based perspectives," IEEE Control Syst. Mag., vol. 29, no. 4, pp. 28-59, Aug. 2009.

[10] D. Jeltsema and A. J. van der Schaft, "Memristive port-Hamiltonian systems," Math. Comput. Model. Dyn. Syst., vol. 16, no. 2, pp. 75-93, Apr. 2010.

[11] G. F. Oster and D. M. Auslander, "The memristor: A new bond graph element," Trans. ASME Dyn. Syst. Control, vol. 94, no. 3, pp. 249-252, 1973.
[12] Y. V. Pershin, S. La Fontaine, and M. Di Ventra, "Memristive model of amoeba's learning," Phys. Rev. E, vol. 80, 021926, 2009.

[13] M. C. Smith, "Synthesis of mechanical networks: The inerter," IEEE Trans. Autom. Control, vol. 47, no. 10, pp. 1648-1662, Oct. 2002.

[14] D. B. Strukov, G. S. Snider, D. R. Stewart, and R. S. Williams, "The missing memristor found," Nature, vol. 453, pp. 80-83, 2008.

[15] A. Stulov, "Hysteretic model of the grand piano hammer felt," J. Acoust. Soc. Amer. vol. 97, no. 4, pp. 2577-2585, 1995.

[16] R. Süßse, P. Burger, U. Diemar, T. Ströhla, and B. Marx, Theoretische Grundlagen der Elektrotechnik 1, B. G. Tuebner, Ed. Wiesbaden, Germany: Verlag/GWV Fachlage $\mathrm{GmbH}, 2005$.

[17] A. J. van der Schaft, $\mathcal{L}_{2}$-Gain and Passivity Techniques in Nonlinear Control. New York: Springer-Verlag, 2000.

\section{ABOUT THE AUTHORS}

Dimitri Jeltsema received the B.Eng. degree in electrical engineering from the Rotterdam School of Engineering, Rotterdam, The Netherlands, in 1996, the M.Sc. degree in systems and control engineering from the University of Hertfordshire, Hatfield, U.K., in 2000, and the Ph.D. degree in systems and control (with honors) from Delft University of Technology, Delft, The Netherlands, in 2005.

From 2005 to 2007, he was a Postdoctoral

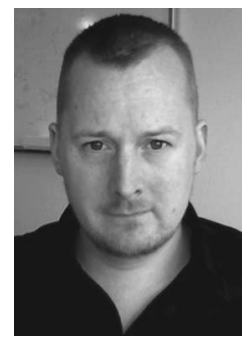
Researcher and Lecturer at the Delft Center for Systems and Control. Currently, he is an Assistant Professor at the Optimization and Systems Theory Group of the Delft Institute of Applied Mathematics, Delft University of Technology. His research interests include modeling, analysis, and control of nonlinear circuits and systems.
Arnau Dòria-Cerezo received the undergraduate degree in electromechanical engineering and the Ph.D. degree in systems and control from the Universitat Politècnica de Catalunya (UPC), Spain, in 2001 and 2006, respectively. He received the DEA in industrial automation from Laboratoire d'Automatisme Industrielle, INSA-Lyon, France, in 2001.

He is currently a tenure-track Professor in the Department of Electrical Engineering, UPC, and carries out his research at the Institut d'Organització i Control de Sistemes Industrials. His research interests include modeling, and advanced control of electrical machines and power converters. 